\title{
On Type II Half Logistic Weibull Distribution with Applications
}

\author{
Amal S. Hassan ${ }^{1}$, M. Elgarhy ${ }^{2}$, Muhammad Ahsan ul Haq ${ }^{3}$, and Sharifah Alrajhi ${ }^{4}$ \\ ${ }^{1}$ Institute of Statistical Studies and Research (ISSR), Cairo University, Department of Mathematical Statistics, \\ Egypt \\ ${ }^{2}$ Vice Presidency for Graduate Studies and Scientific Research, University of Jeddah, Jeddah, KSA \\ ${ }^{3}$ College of Statistical \& Actuarial Sciences, University of the Punjab, Lahore, Pakistan \\ Quality Enhancement Cell, National College of Arts, Lahore-Pakistan \\ ${ }^{4}$ Statistics Department, Faculty of Science, King AbdulAziz University, Jeddah, Kingdom of Saudi Arabia \\ E-mails : dr.amalelmoslamy@gmail.com,m_elgarhy85@yahoo.com, ahsanshani36@gmail.com, and \\ saalrajhi@kau.edu.sa
}

\begin{abstract}
In recent years, several of new improved and extended probability distributions have been discovered from the current distributions to facilitate their applications in many fields. A new three-parameter distribution, the so called the Type II half logistic Weibull (TIIHLW), is introduced for modeling lifetime data. Some mathematical properties of the TIIHLW distribution are provided. Explicit expressions for the moments, probability weighted moments, quantile function, order statistics and Rényi entropy are investigated. Maximum likelihood estimation technique is employed to estimate the model parameters and simulation issues are presented. In addition, the superiority of the subject distribution is illustrated with an application to two real data sets. Indeed, the TIIHLW model yields a better fit to these data than the beta Weibull, Mcdonald Weibull and exponentiated Weibull distributions.
\end{abstract}

Keywords: Type II half logistic-G class; Weibull distribution, Order statistics; Maximum likelihood method.

DOI: $10.7176 / \mathrm{MTM} / 9-1-05$

\section{Introduction}

The Weibull (W) distribution is a very popular distribution for modeling lifetime data in reliability where the hazard rate function is monotone. However, in many applied areas, the two-parameter $\mathrm{W}$ distribution is inadequate for modeling phenomenon with non-monotone hazard rate. Various generalizations and extensions of the W distribution have been proposed in the statistical literature to handle with bathtub shaped failure rates. Mudholkar and Srivastava (1993) and Mudholkar et al. (1996) pioneered exponentiated $W(\mathrm{EW})$ distribution to analyze bathtub failure data. Xie et al. (2002) proposed a three- parameter modified W extension with a bathtub shaped hazard function. Carrasco et al. (2008) suggested the generalized modified W distribution, among others.

Recently, new generated families of continuous distributions have been attracted several statisticians to develop new models. These families are obtained by introducing one or more additional shape parameter(s) to the baseline distribution. Some of the generated families are: the beta-G (Eugene et al. (2002)), gamma-G (Zografos and Balakrishanan (2009)), Kumaraswamy-G (Cordeiro and de Castro (2011)), McDonald-G (Alexander et al. (2012)), transformed-transformer (Alzaatreh et al. (2013)), Kumaraswamy odd log-logistic (Alizadeh et al. (2015)), Type 1 half-logistic family (Cordeiro et al. (2015)), Garhy generated family (Elgarhy et al. (2016)), Kumaraswamy Weibull-G (Hassan and Elgarhy (2016a)), exponentiated Weibull-generated family (Hassan and Elgarhy (2016b)), additive Weibull-G (Hassan and Hemeda (2016)), Type II half logistic-G(TIIHL-G) (Hassan et al. (2017a)), generalized additive Weibull-G by (Hassan et al. (2017 b)), and power Lindley-G (Hassan and Nassr (2018))among others.

The cumulative distribution function (cdf) of the TIIHL-G family is given by

$$
F(x ; \lambda, \zeta)=\frac{2[G(x ; \zeta)]^{\lambda}}{1+[G(x ; \zeta)]^{\lambda}}, x>0, \lambda>0,
$$

where $\lambda$ is the shape parameter. The probability density function (pdf) corresponding to (1) is given by 


$$
f(x ; \lambda, \zeta)=\frac{2 \lambda g(x ; \zeta)[G(x ; \zeta)]^{\lambda-1}}{\left[1+[G(x ; \zeta)]^{\lambda}\right]^{2}}, x>0, \lambda>0 .
$$

Our motivation here is to extend the two- parameter Weibull distribution to produce a more flexible model. The new model is referred to as the Type II half logistic Weibull distribution. Based on the TIIHL-G family, we construct the TIIHLW distribution as well as we provide the main statistical distributions. The remainder of the paper is organized as follows: In Section 2, we define the TIIHLW distribution and provide its special models. In Section 3, we derive a very useful representation for the TIIHLW density and distribution functions. Further, we derive some mathematical properties of the subject distribution. The maximum likelihood method is used to estimate the model parameters in Section 4. In Section 5, simulation study is conducted to assess the performance of model parameters. In Section 6, we demonstrate the importance of the TIIHLW distribution using two real data sets. Finally, we give some concluding remarks in Section 7.

\section{Type II Half Logistic Weibull Distribution}

The cdf of the W distribution with scale parameter $\delta>0$ and shape parameter $\gamma>0$ is given (for $x>0$ ) by

$$
G(x ; \delta, \gamma)=1-\mathrm{e}^{-\delta x^{\gamma}} \text {. }
$$

The pdf corresponding to (3) is given by

$$
g(x ; \delta, \gamma)=\delta \gamma x^{\gamma-1} \mathrm{e}^{-\delta x^{\gamma}}
$$

The random variable $X$ is said to have a TIIHLW distribution, denoted by $X \sim$ TIIHLW $(\lambda, \delta, \gamma)$, if its cdf is obtained by inserting cdf (3) in cdf (1) as follows

$$
F(x ; \lambda, \delta, \gamma)=\frac{2\left[1-e^{-\delta x^{\gamma}}\right]^{\lambda}}{1+\left[1-e^{-\delta x^{\gamma}}\right]^{\lambda}} ; \quad \lambda, \delta, \gamma>0, x>0 \text {. }
$$

The pdf corresponding to (5) is as follows

$$
f(x ; \lambda, \delta, \gamma)=\frac{2 \lambda \delta \gamma x^{\gamma-1} e^{-\delta x^{\gamma}}\left[1-e^{-\delta x^{\gamma}}\right]^{\lambda-1}}{\left[1+\left[1-e^{-\delta x^{\gamma}}\right]^{\lambda}\right]^{2}}
$$

For $\gamma=1$, the pdf (6) reduces to a new model called TIIHL- exponential distribution and for $\gamma=2$, the pdf (6) reduces to another new model called TIIHL- Rayleigh distribution. The pdf plots for the TIIHLW are presented in Figure 1. As seen from Figure 1, densities of TIIHLW distribution take different shapes like, symmetric, right skewed, reversed $\mathbf{J}$ shaped and unimodel. 


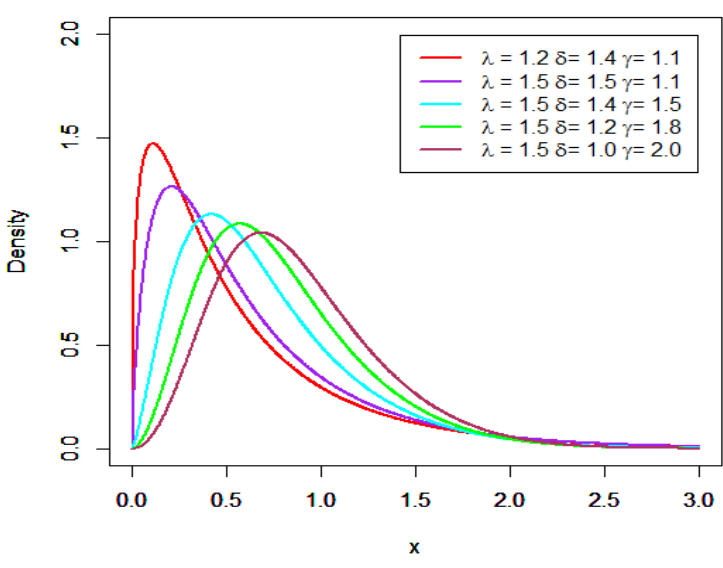

(a)

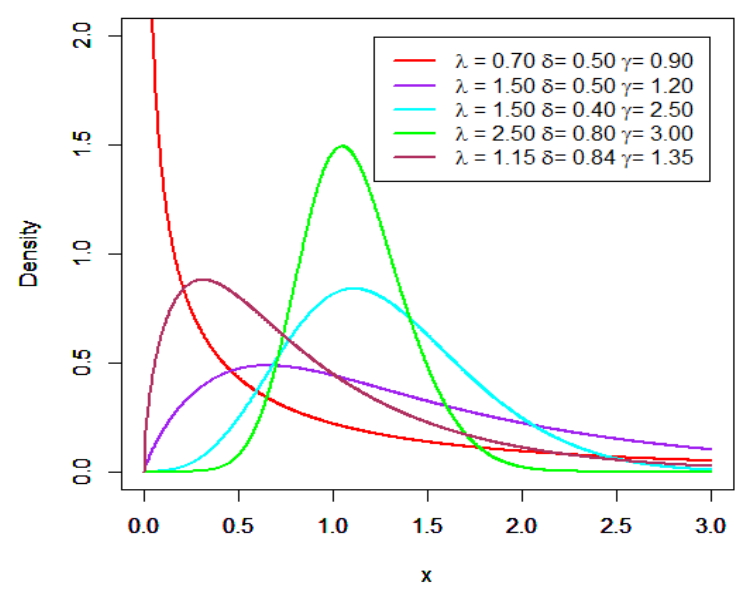

(b)

Figure 1: The pdf of TIIHLW distribution for different values of parameters

Further, the survival function of $X$, denoted byis as follows $\bar{F}(x ; \lambda, \delta, \gamma)$,

$$
\bar{F}(x ; \lambda, \delta, \gamma)=\frac{1-\left[1-e^{-\delta x^{\gamma}}\right]^{\lambda}}{1+\left[1-e^{-\delta x^{\gamma}}\right]^{\lambda}} .
$$

Additionally, the hazard rate function (hrf), saycan be written as follows $h(x ; \lambda, \delta, \gamma)$,

$$
h(x ; \lambda, \delta, \gamma)=\frac{2 \lambda \delta \gamma x^{\gamma-1} e^{-\delta x^{\gamma}}\left[1-e^{-\delta x^{\gamma}}\right]^{\lambda-1}}{1-\left[1-e^{-\delta x^{\gamma}}\right]^{2 \lambda}} .
$$

The hrf plots for the TIIHLW are displayed in Figure 2. It is clear from Figure 2 that the hrf plots takes different shapes according to different values of parametrs. It can be increasing, decreasing, up-side down and U-shaped.

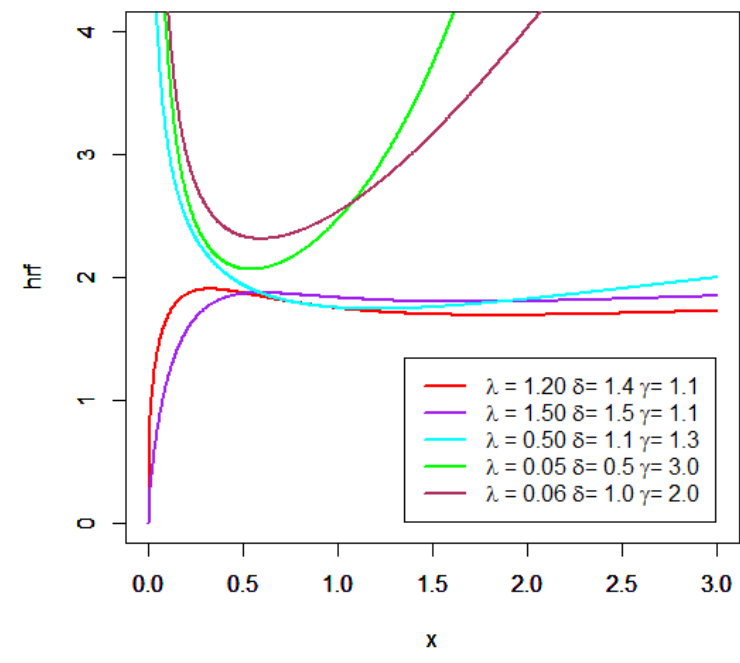

(a)

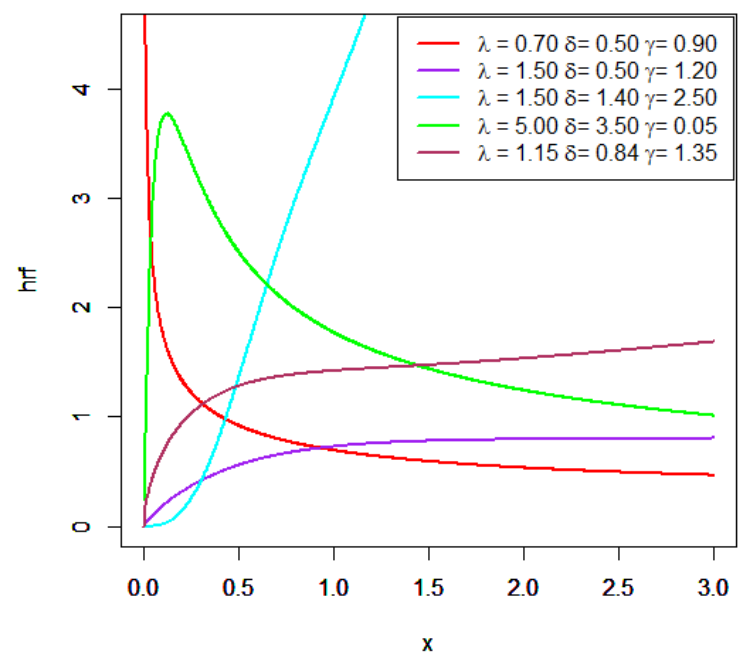

(b)

Figure 2: The hrf of TIIHLW distribution for different values of parameters 


\section{Some Statistical Properties}

This section provides some statistical properties of TIIHLW distribution.

\subsection{Quantile function}

The quantile function of the TIIHLW distribution is obtained by inverting cdf (5) as follows

$$
X_{Q}=\left\{\frac{-1}{\delta} \ln \left(1-\left[\frac{Q}{2-Q}\right]^{\frac{1}{\lambda}}\right)\right\}^{\frac{1}{\gamma}}, \quad 0<Q<1 .
$$

Specifically, the first quartile, the median, and the third quartile are obtained by setting $Q=0.25,0.5$ and 0.75 , respectively, in (7). Also, the random variable $X$ has TIIHLW distribution can be generated from (7), where $Q$ has the uniform distribution over the interval $(0,1)$. Furthermore, the analysis of the variability of the skewness and kurtosis on the shape parameters $\lambda$ and $\gamma$ can be investigated based on quantile measures. The Bowley skewness (see Kenney and Keeping (1962)), denoted by $B$, is defined by

$$
B=\frac{X_{3 / 4}-2 X_{1 / 2}+X_{1 / 4}}{X_{3 / 4}-X_{1 / 4}} .
$$

The Moors kurtosis (see Moors (1988)), denoted by $M$, can be defined as follows

$$
M=\frac{X_{7 / 8}-X_{5 / 8}+X_{3 / 8}-X_{1 / 8}}{X_{6 / 8}-X_{2 / 8}} .
$$

The Bowley skewness and Moors kurtosis measures do not depend on the moments of the distribution and are almost insensitive to outliers. Plots of the skewness and kurtosis for some choices of the parameter $\lambda$ as function of $\gamma$, and for some choices of the parameter $\gamma$ as function of $\lambda$ are shown in Figures 3 and 4 . We can reveal from these figures that the skewness and kurtosis for $\lambda$ decrease as $\gamma$ increases from 0.5 to 2.5 for fixed value of $\delta=0.5$. Also, the skewness and kurtosis for $\gamma$ decrease as $\lambda$ increases from 0.5 to 3 for fixed value of $\delta=0.5$

(a)

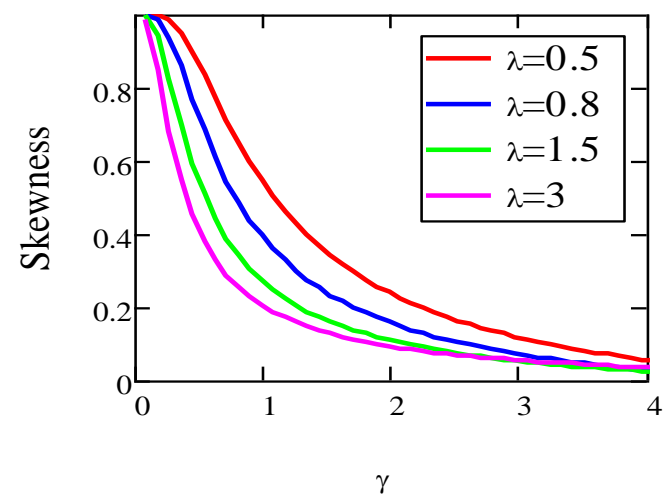

(b)

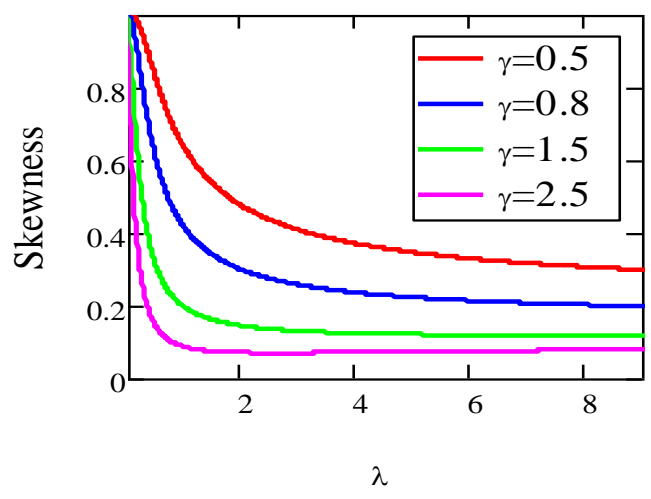

Figure 3: Bowley skewness of the TIIHLW distribution. (a) As function of $\gamma$ for some values of $\lambda$ (b) As function of $\lambda$ for some values of $\gamma$ 
(a)

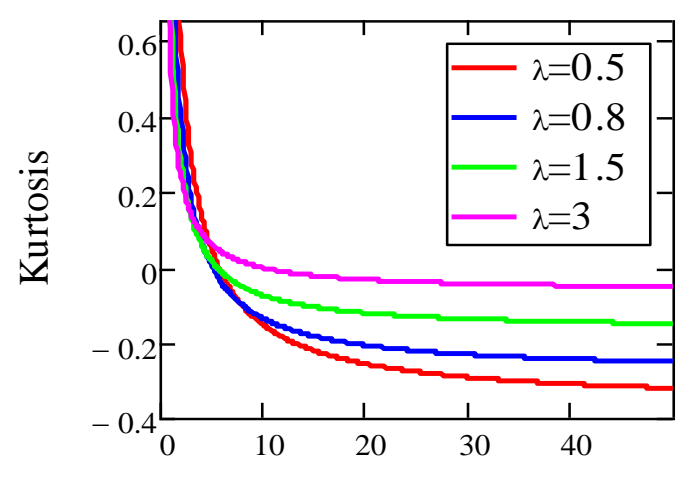

$\gamma$ (b)

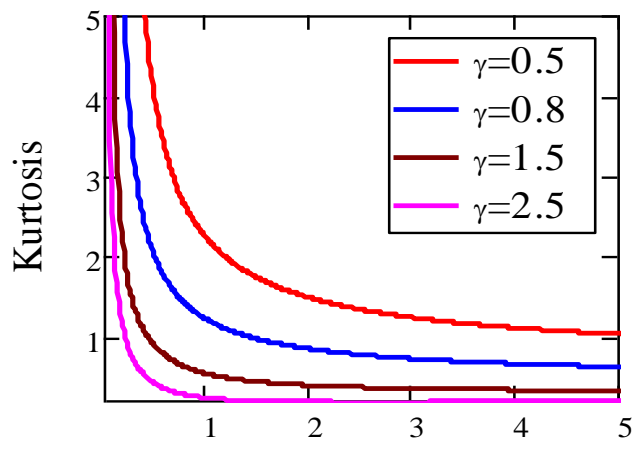

B

Figure 4: Moors kurtosis of the TIIHLW distribution. (a) As function of $\gamma$ for some values of $\lambda$ (b) As function of $\lambda$ for some values of $\gamma$

\subsection{Important Representation}

The pdf and cdf expansions of TIIHLW are provided, which are useful in studying most statistical properties of TIIHLW distribution. From a generalized binomial series, it is known that, for $|z|<1$, and $\beta$ is a positive real non integer,

$$
(1+z)^{-\beta}=\sum_{i=0}^{\infty}(-1)^{i}\left(\begin{array}{c}
\beta+i-1 \\
i
\end{array}\right) z^{i}
$$

Then, by applying the binomial theorem (8) in pdf (6), then, we have

$$
f(x ; \lambda, \delta, \gamma)=\sum_{i=0}^{\infty} \eta_{i} x^{\gamma-1} e^{-\delta x^{\gamma}}\left[1-e^{-\delta x^{\gamma}}\right]^{\lambda(i+1)-1},
$$

where, $\eta_{i}=2 \lambda \delta \gamma(-1)^{i}(i+1)$. Now, using the generalized binomial theorem, we can write

$$
\left[1-e^{-\delta x^{\gamma}}\right]^{\lambda(i+1)-1}=\sum_{j=0}^{\infty}(-1)^{j}\left(\begin{array}{c}
\lambda(i+1)-1 \\
j
\end{array}\right) e^{-j \delta x^{\gamma}} .
$$

Inserting the expansion (11) in (10), then the pdf (8) will be converted to

$$
\begin{gathered}
f(x ; \lambda, \delta, \gamma)=\sum_{i, j=0}^{\infty} \eta_{i, j} x^{\gamma-1} e^{-\delta(j+1) x^{\gamma}}, \\
\eta_{i, j}=(-1)^{j}\left(\begin{array}{c}
\lambda(i+1)-1 \\
j
\end{array}\right) \eta_{i} .
\end{gathered}
$$

where,

Further, an extra expansion for the $[F(x ; \lambda, \delta, \gamma)]^{s}$, for $s$ an integer, is derived, again the binomial expansion is worked out;

$$
[F(x ; \lambda, \delta, \gamma)]^{s}=\sum_{k=0}^{s} 2^{s}(-1)^{k}\left(\begin{array}{c}
s+k-1 \\
k
\end{array}\right)\left[1-e^{-\delta x^{\gamma}}\right]^{\lambda(k+s)} .
$$


The binomial expansion is employed another time to the last equation, then $[F(x ; \lambda, \delta, \gamma)]^{s}$, can be reformed as follows

$$
[F(x ; \lambda, \delta, \gamma)]^{s}=\sum_{k=0}^{s} \sum_{l=0}^{\infty} 2^{s}(-1)^{k+l}\left(\begin{array}{c}
s+k-1 \\
k
\end{array}\right)\left(\begin{array}{c}
\lambda(k+s) \\
l
\end{array}\right) e^{-\delta l x^{\gamma}}
$$

For $s$ is a real, then $[F(x ; \lambda, \delta, \gamma)]^{s}$, is as follows

$$
[F(x ; \lambda, \delta, \gamma)]^{s}=\sum_{k=0}^{\infty} \sum_{l=0}^{\infty} \eta_{k, l} e^{-\delta l x^{\gamma}}
$$

where,

$$
\eta_{k, l}=2^{s}(-1)^{k+l}\left(\begin{array}{c}
s+k-1 \\
k
\end{array}\right)\left(\begin{array}{c}
\lambda(k+s) \\
l
\end{array}\right) .
$$

\subsection{Probability Weighted Moments}

Class of moments, called the probability-weighted moments (PWMs), has been proposed by Greenwood et al. (1979). This class is used to derive estimators of the parameters and quantiles of distributions expressible in inverse form. For a random variable $X$, the PWMs, denoted by $\tau_{r, s}$, can be calculated according to the following relation

$$
\tau_{r, s}=E\left[X^{r} \mathrm{~F}(x)^{s}\right]=\int_{-\infty}^{\infty} x^{r} \mathrm{f}(x)(\mathrm{F}(x))^{s} d x
$$

Inserting (11) and (12) in (13), the PWMs of TIIHLW will be converted to

$$
\tau_{r, s}=\sum_{i, j, l=0}^{\infty} \sum_{k=0}^{s} \eta_{i, j} \eta_{k, l} \int_{0}^{\infty} x^{r+\gamma-1} e^{-\delta(l+j+1) x^{\gamma}} d x
$$

So, after some simplifications, the PWMs of TIIHLW can be written as follows

$$
\tau_{r, s}=\sum_{i, j, l=0}^{\infty} \sum_{k=0}^{s} \frac{\eta_{i, j} \eta_{k, l} \Gamma(r / \gamma+1)}{\gamma[\delta(l+j+1)]^{\frac{r}{\gamma}+1}}
$$

\subsection{Moments}

In this subsection we derive the $r^{\text {th }}$ moment for the TIIHLW distribution. If $X$ has the pdf (11), then $r^{\text {th }}$ moment is obtained as follows

$$
\mu_{r}^{\prime}=\sum_{i, j=0}^{\infty} \eta_{i, j} \int_{0}^{\infty} x^{r+\gamma-1} e^{-\delta(j+1) x^{\gamma}} d x=\sum_{i, j=0}^{\infty} \frac{\eta_{i, j} \Gamma(r / \gamma+1)}{\gamma[\delta(j+1)]^{\frac{r}{\gamma}+1}}
$$

The mean and variance of TIIHLW distribution are as follows

$$
E(X)=\sum_{i, j=0}^{\infty} \frac{\eta_{i, j} \Gamma(1 / \gamma+1)}{\gamma[\delta(j+1)]^{\frac{1}{\gamma}+1}}
$$

and, 


$$
\operatorname{var}(X)=\sum_{i, j=0}^{\infty} \frac{\eta_{i, j} \Gamma(2 / \gamma+1)}{\gamma[\delta(j+1)]^{\frac{2}{\gamma}+1}}-\left[\sum_{i, j=0}^{\infty} \frac{\eta_{i, j} \Gamma(1 / \gamma+1)}{\gamma[\delta(j+1)]^{\frac{1}{\gamma}+1}}\right]^{2}
$$

Figure 5 illustrates the mean and variance whose forms depend basically on the parameters $\gamma$ and $\delta$.

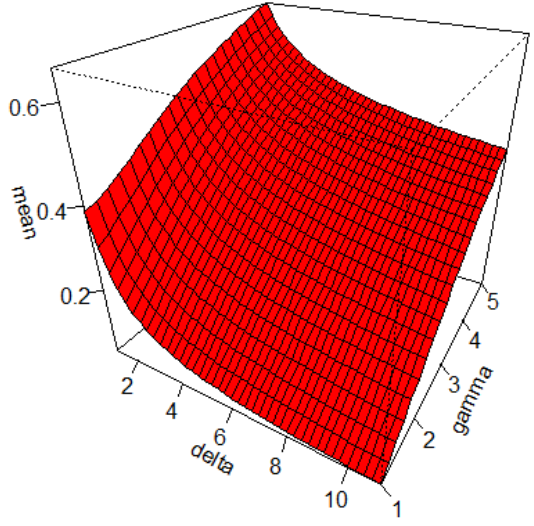

mean

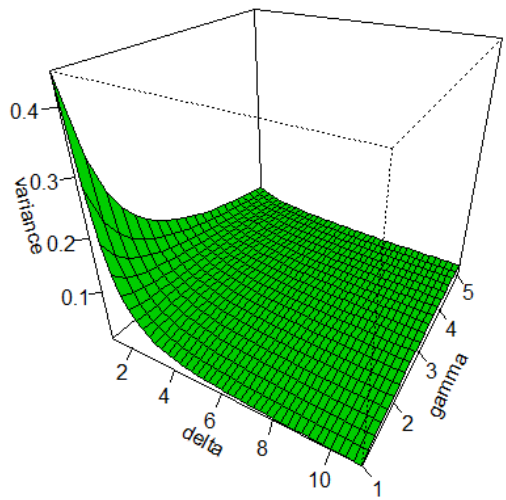

variance

Figure 5: Mean and Variance of TIIHLW distribution for selected values of parameters

Furthermore, for a random variable $X$, the moment generating function of TIIHLW distribution is given by

$$
M_{X}(t)=\sum_{r=0}^{\infty} \frac{t^{r}}{r !} \mu_{r}^{\prime}=\sum_{r, i, j=0}^{\infty} \frac{\eta_{i, j} t^{r} \Gamma(r / \gamma+1)}{\gamma r ![\delta(j+1)]^{\frac{r}{\gamma}+1}} .
$$

\subsection{Order Statistics}

Order statistics have been extensively applied in many fields of statistics, such as reliability and life testing. Let $X_{1}, X_{2}, \ldots, X_{n}$ be independent and identically distributed random variables with their corresponding continuous distribution function $F(x)$. Let $X_{(1)}<X_{(2)}<\ldots<X_{(n)}$ be the corresponding ordered random sample from a population of size $n$. According to David (1981), the pdf of the $r^{\text {th }}$ order statistic, is defined as

$$
f_{X_{(r)}}(x)=\frac{f(x)}{B(r, n-r+1)} \sum_{v=0}^{n-r}(-1)^{v}\left(\begin{array}{c}
n-r \\
v
\end{array}\right) F(x)^{v+r-1},
$$

where, $B(.,$.$) is the beta function. The pdf of the r^{\text {th }}$ order statistic for TIIHLW distribution is derived by substituting (11) and (12) in (14), replacing $s$ with $v+r-1$,

$$
\begin{gathered}
f_{X_{(r)}}(x)=\frac{1}{B(r, n-r+1)} \sum_{v=0 i, j, l=0}^{n-r} \sum_{k=0}^{\infty} \sum^{v+r-1} \eta^{*} x^{\gamma-1} e^{-\delta(l+j+1) x^{\gamma}}, \\
\eta^{*}=(-1)^{k+l+v} 2^{v+r-1}\left(\begin{array}{c}
n-r \\
v
\end{array}\right)\left(\begin{array}{c}
v+r+k-2 \\
k
\end{array}\right)\left(\begin{array}{c}
\lambda(k+v+r-1) \\
l
\end{array}\right) \eta_{i, j} .
\end{gathered}
$$

where,

The distribution of the smallest and largest order statistics can be obtained individually from (15) by setting $r=1$ and $r=n$. Further, the $k^{\text {th }}$ moment of $r^{\text {th }}$ order statistics for TIIHLW distribution is defined by: 


$$
E\left(X_{(r)}{ }^{k}\right)=\int_{-\infty}^{\infty} x^{k} f_{X_{(r)}}(x ; \lambda, \delta, \gamma) d x .
$$

By substituting (15) in (16), leads to

$$
E\left(X_{(r)}{ }^{k}\right)=\frac{1}{B(r, n-r+1)} \sum_{v=0 i, j, l=0}^{n-r} \sum_{k=0}^{\infty} \sum_{k[\delta(l+j+1)]^{\frac{k}{\gamma}+1}} .
$$

\subsection{Rényi Entropy}

The entropy of a random variable $X$ is a measure of variation of uncertainty. It has been used in many fields such as physics, engineering and economics. According to Rényi (1961), the Rényi entropy is defined by

$$
I_{\alpha}(X)=\frac{1}{1-\alpha} \log \int_{-\infty}^{\infty} f(x)^{\alpha} d x, \quad \alpha>0 \text { and } \quad \alpha \neq 1 .
$$

By applying the binomial theory (8) and (10) in pdf $f(x ; \lambda, \delta, \gamma)^{\alpha}$, then it can be expressed as follows

$$
f(x ; \lambda, \delta, \gamma)^{\alpha}=\sum_{i, j=0}^{\infty} t_{i, j} x^{\alpha(\gamma-1)} e^{-\delta(\alpha+j) x^{\gamma}},
$$

where,

$$
t_{i, j}=(2 \lambda \delta \gamma)^{\alpha}(-1)^{i+j}\left(\begin{array}{c}
2 \alpha+i-1 \\
i
\end{array}\right)\left(\begin{array}{c}
\lambda(\alpha+i)-\alpha \\
j
\end{array}\right) .
$$

Therefore, the Rényi entropy of TIIHLW distribution is given by

$$
I_{\alpha}(X)=\frac{1}{1-\alpha} \log \left[\sum_{i, j=0}^{\infty} \frac{t_{i, j} \Gamma\left(\alpha-\frac{\alpha}{\gamma}+\frac{1}{\gamma}\right)}{\gamma[\delta(\alpha+j)]^{\alpha-\frac{\alpha}{\gamma}+\frac{1}{\gamma}}}\right]
$$

\subsection{Stress-Strength Reliability}

Let $X_{1}$ be the strength of a system which is subjected to a stress $X_{2}$, and if $X_{1}$ follows TIIHLW $\left(\lambda_{1}, \delta_{1}, \gamma\right)$ and $X_{2}$ follows TIIHLW $\left(\lambda_{2}, \delta_{2}, \gamma\right)$, provided $X_{1}$ and $X_{2}$ are statistically independent random variables, then $R=P\left(X_{2}<\right.$ $X_{1}$ ), the measure of system performance (stress strength reliability measure) is given by,

$$
\begin{aligned}
& R=P\left(X_{2}<X_{1}\right)=\int_{0}^{\infty} f_{1}(x) F_{2}(x) d x, \\
& R=\int_{0}^{\infty} \frac{2 \lambda_{1} \delta_{1} \gamma x x^{\gamma-1} e^{-\delta_{1} x^{\gamma}}\left[1-e^{-\delta_{1} x^{\gamma}}\right]^{\lambda_{1}-1}}{\left[1+\left[1-e^{-\delta_{1} x^{\gamma}}\right]^{\lambda_{1}}\right]^{2}} \times \frac{2\left[1-e^{-\delta_{2} x^{\gamma}}\right]^{\lambda_{2}}}{1+\left[1-e^{-\delta_{2} x^{\gamma}}\right]^{\lambda_{2}}} d x .
\end{aligned}
$$

Applying the series expansion (8), on the last two terms of the integrand with some mathematical manipulations, we can write the previous equation as 
where

$$
R=\sum_{i, j, k, l=0}^{\infty} \eta_{i, j, k, l} \int_{0}^{\infty} x^{\gamma-1} e^{-\left(\delta_{2} l+(j+1) \delta_{1}\right) x^{\gamma}} d x
$$

$$
\eta_{i, j, k, l}=(-1)^{i+j+k+l} 4 \lambda_{1} \delta_{1} \gamma(-1)^{i}(i+1)\left(\begin{array}{c}
\lambda_{1}(i+1)-1 \\
j
\end{array}\right)\left(\begin{array}{c}
\lambda_{2}(k+1) \\
l
\end{array}\right) .
$$

Then, we get, finally, the form of $\mathrm{R}$ as

$$
R=\sum_{i, j, k, l=0}^{\infty} \frac{\eta_{i, j, k, l}}{\gamma\left[\delta_{2} l+(j+1) \delta_{1}\right]}
$$

\section{Maximum Likelihood Method}

This section deals with the maximum likelihood estimators of the unknown parameters for the TIIHLW distribution on the basis of complete samples. Let $X_{1}, \ldots, X_{n}$ be the observed values from the TIIHLW distribution with set of parameter $\Phi=(\lambda, \delta, \gamma)^{T}$. The log-likelihood function for parameter vector $\Phi=(\lambda, \delta, \lambda)^{T}$ is obtained as follows

$$
\begin{aligned}
\ln L(\Phi)= & n \ln 2 \lambda+n \ln \delta+n \ln \gamma+(\gamma-1) \sum_{i=1}^{n} \ln \left(x_{i}\right)-\delta \sum_{i=1}^{n} x_{i}^{\gamma} \\
& +(\lambda-1) \sum_{i=1}^{n} \ln \left[1-e^{-\delta x_{i}^{\gamma}}\right]-2 \sum_{i=1}^{n} \ln \left[1+\left[1-e^{-\delta x_{i}^{\gamma}}\right]^{\lambda}\right]
\end{aligned}
$$

The elements of the score function $U(\Phi)=\left(U_{\lambda}, U_{\delta}, U_{\gamma}\right)$ are given by

$$
\begin{gathered}
U_{\lambda}=\frac{n}{\lambda}+\sum_{i=1}^{n} \ln \left[1-e^{-\delta x_{i}{ }^{\gamma}}\right]-2 \sum_{i=1}^{n} \frac{\ln \left[1-e^{-\delta x_{i}{ }^{\gamma}}\right]}{\left[\left[1-e^{-\delta x_{i}{ }^{\gamma}}\right]^{-\lambda}-1\right]}, \\
U_{\delta}=\frac{n}{\delta}-\sum_{i=1}^{n} x_{i}^{\gamma}+(\lambda-1) \sum_{i=1}^{n} \frac{x_{i}^{\gamma}}{e^{\delta x_{i}{ }^{\gamma}}-1}-2 \lambda \sum_{i=1}^{n} \frac{x_{i}^{\gamma} e^{-\delta x_{i}{ }^{\gamma}}\left[1-e^{-\delta x_{i}{ }^{\gamma}}\right]^{\lambda-1}}{1+\left[1-e^{-\delta x_{i}{ }^{\gamma}}\right]^{\lambda}},
\end{gathered}
$$

and

$$
U_{\gamma}=\frac{n}{\gamma}+\sum_{i=1}^{n} \ln \left(x_{i}\right)-\delta \sum_{i=1}^{n} x_{i}{ }^{\gamma} \ln x_{i}+\delta(\lambda-1) \sum_{i=1}^{n} \frac{x_{i}{ }^{\gamma} \ln x_{i}}{e^{\delta x_{i}{ }^{\gamma}}-1}-2 \lambda \delta \sum_{i=1}^{n} \frac{\left[1-e^{-\delta x_{i}{ }^{\gamma}}\right]^{\lambda-1} x_{i}{ }^{\gamma} \ln x_{i} e^{-\delta x_{i}{ }^{\gamma}}}{1+\left[1-e^{-\delta x_{i}{ }^{\gamma}}\right]^{\lambda}} .
$$

Setting $U_{\lambda}, U_{\delta}$ and $U_{\gamma}$ equal to zero and solving these equations simultaneously yield the maximum likelihood estimate (MLE) $\hat{\Phi}=(\hat{\lambda}, \hat{\delta}, \hat{\gamma})$ of $\Phi=(\lambda, \delta, \gamma)^{T}$. These equations cannot be solved analytically and statistical software can be used to solve them numerically using iterative methods.

\section{Simulation Study}

In this section, an extensive numerical investigation will be carried out to evaluate the performance of MLE for TIIHLW model. Performance of estimators is evaluated through their biases and mean square errors (MSEs) for 
different sample sizes. A numerical study is performed using Mathematica (7) software. The simulation procedure is worked out as follows:

Step (1): 10000 random samples of sizes 10, 20, 30, 50, 100,200 and 300 are generated from TIIHLW distribution.

Step (2): Different values of parameters $(\lambda, \delta, \gamma)$ are selected as $(0.5,0.5,0.9),(0.5,0.9,0.5),(0.5,0.5,1.2)$ and $(0.5,1.2,0.5)$.

Step (3): For each sample size and for each set of parameters MLE of the parameters $\hat{\lambda}, \hat{\delta}$ and $\hat{\gamma}$ are obtained by iterative technique.

Step (4): The biases and MSE for each sample size are calculated.

Numerical results are listed in Tables 1 and 2. The values in the mentioned tables show that, in general, the mean square error for the estimates of the parameters $\hat{\lambda}, \hat{\delta}$ and $\hat{\gamma}$ decreases as the sample size increases.

Table 1: MLEs, biases and MSEs for some parameter values

\begin{tabular}{|c|c|c|c|c|c|c|c|}
\hline \multirow[b]{2}{*}{$n$} & \multirow[b]{2}{*}{ Parameter } & \multicolumn{3}{|c|}{$(0.5,0.5,0.9)$} & \multicolumn{3}{|c|}{$(0.5,0.9,0.5)$} \\
\hline & & $\begin{array}{c}\text { Estimated } \\
\text { values }\end{array}$ & Bias & MSE & $\begin{array}{c}\text { Estimated } \\
\text { values }\end{array}$ & Bias & MSE \\
\hline \multirow{3}{*}{10} & $\lambda$ & 0.5857 & 0.0857 & 0.1025 & 0.5541 & 0.0541 & 0.0669 \\
\hline & $\delta$ & 1.0677 & 0.1677 & 0.4111 & 0.6570 & 0.1570 & 0.8764 \\
\hline & $\gamma$ & 0.5129 & 0.0129 & 0.0070 & 0.9441 & 0.4441 & 0.2174 \\
\hline \multirow{3}{*}{20} & $\lambda$ & 0.5239 & 0.0239 & 0.0226 & 0.5267 & 0.0267 & 0.0203 \\
\hline & $\delta$ & 0.9771 & 0.0771 & 0.0817 & 0.5280 & 0.0280 & 0.0240 \\
\hline & $\gamma$ & 0.5136 & 0.0136 & 0.0034 & 0.9142 & 0.4142 & 0.1846 \\
\hline \multirow{3}{*}{30} & $\lambda$ & 0.5146 & 0.0146 & 0.0172 & 0.5268 & 0.0268 & 0.0142 \\
\hline & $\delta$ & 0.9656 & 0.0656 & 0.0825 & 0.5402 & 0.0402 & 0.0191 \\
\hline & $\gamma$ & 0.5150 & 0.0150 & 0.0030 & 0.9146 & 0.4146 & 0.1803 \\
\hline \multirow{3}{*}{50} & $\lambda$ & 0.5126 & 0.0126 & 0.0067 & 0.5157 & 0.0157 & 0.0067 \\
\hline & $\delta$ & 0.9267 & 0.0267 & 0.0212 & 0.5185 & 0.0185 & 0.0095 \\
\hline & $\gamma$ & 0.5049 & 0.0049 & 0.0013 & 0.9032 & 0.4032 & 0.1677 \\
\hline \multirow{3}{*}{100} & $\lambda$ & 0.5115 & 0.0115 & 0.0033 & 0.5076 & 0.0076 & 0.0034 \\
\hline & $\delta$ & 0.9221 & 0.0221 & 0.0171 & 0.5094 & 0.0094 & 0.0044 \\
\hline & $\gamma$ & 0.4992 & -0.0009 & 0.0008 & 0.9041 & 0.4041 & 0.1664 \\
\hline \multirow{3}{*}{200} & $\lambda$ & 0.5057 & 0.0057 & 0.0016 & 0.5039 & 0.0039 & 0.0019 \\
\hline & $\delta$ & 0.9153 & 0.0153 & 0.0065 & 0.5079 & 0.0079 & 0.0024 \\
\hline & $\gamma$ & 0.5011 & 0.0011 & 0.0004 & 0.9049 & 0.4049 & 0.1654 \\
\hline \multirow{3}{*}{300} & $\lambda$ & 0.5007 & 0.0007 & 0.0012 & 0.5032 & 0.0032 & 0.0008 \\
\hline & $\delta$ & 0.9002 & 0.0002 & 0.0054 & 0.5054 & 0.0054 & 0.0011 \\
\hline & $\gamma$ & 0.5002 & 0.0002 & 0.0002 & 0.9012 & 0.4012 & 0.1617 \\
\hline
\end{tabular}


Table 2: MLEs, biases and MSEs for some parameter values

\begin{tabular}{cccccccc}
\hline & \multicolumn{3}{c}{$(0.5,0.5,1.2)$} & \multicolumn{3}{c}{$(0.5,1.2,0.5)$} \\
\hline $\boldsymbol{n}$ & Parameter & $\begin{array}{c}\text { Estimated } \\
\text { values }\end{array}$ & Bias & MSE & $\begin{array}{c}\text { Estimated } \\
\text { values }\end{array}$ & Bias & MSE \\
\hline \multirow{3}{*}{10} & $\lambda$ & 0.5789 & 0.0789 & 0.0740 & 0.5882 & 0.0882 & 0.0795 \\
& $\delta$ & 1.3384 & 0.1384 & 0.2770 & 0.6480 & 0.1480 & 0.2848 \\
& $\gamma$ & 0.5121 & 0.0121 & 0.0087 & 1.2279 & 0.7278 & 0.5708 \\
\hline \multirow{3}{*}{20} & $\lambda$ & 0.5486 & 0.0486 & 0.0279 & 0.5347 & 0.0347 & 0.0316 \\
& $\delta$ & 1.3109 & 0.1109 & 0.1835 & 0.5527 & 0.0527 & 0.0698 \\
& $\gamma$ & 0.5055 & 0.0055 & 0.0042 & 1.2331 & 0.7331 & 0.5628 \\
\hline \multirow{3}{*}{30} & $\lambda$ & 0.5104 & 0.0104 & 0.0136 & 0.5320 & 0.0320 & 0.0171 \\
& $\delta$ & 1.2520 & 0.0520 & 0.0986 & 0.5382 & 0.0382 & 0.0217 \\
& $\gamma$ & 0.5101 & 0.0101 & 0.0030 & 1.2123 & 0.7123 & 0.5255 \\
\hline \multirow{3}{*}{50} & $\lambda$ & 0.5256 & 0.0256 & 0.0070 & 0.5248 & 0.0248 & 0.0085 \\
& $\delta$ & 1.2602 & 0.0602 & 0.0423 & 0.5296 & 0.0296 & 0.0128 \\
& $\gamma$ & 0.4996 & -0.0004 & 0.0016 & 1.2027 & 0.7027 & 0.5045 \\
\hline \multirow{3}{*}{100} & $\lambda$ & 0.5007 & 0.0007 & 0.0047 & 0.5146 & 0.0146 & 0.0041 \\
& $\delta$ & 1.2041 & 0.0041 & 0.0337 & 0.5169 & 0.0169 & 0.0050 \\
& $\gamma$ & 0.5022 & 0.0022 & 0.0008 & 1.2055 & 0.7055 & 0.5036 \\
\cline { 2 - 8 } 200 & $\lambda$ & 0.5004 & 0.0004 & 0.0016 & 0.5006 & 0.0006 & 0.0024 \\
& $\delta$ & 1.2029 & 0.0029 & 0.0132 & 0.5003 & 0.0003 & 0.0030 \\
& $\gamma$ & 0.5003 & 0.0003 & 0.0004 & 1.2043 & 0.7043 & 0.4986 \\
\cline { 2 - 8 } 300 & $\lambda$ & 0.5017 & 0.0017 & 0.0013 & 0.5029 & 0.0029 & 0.0013 \\
& $\delta$ & 1.2066 & 0.0066 & 0.0100 & 0.5035 & 0.0035 & 0.0015 \\
& $\gamma$ & 0.5011 & 0.0011 & 0.0003 & 1.2022 & 0.7022 & 0.4950 \\
\hline
\end{tabular}

\section{Data Analysis}

In this section, we use two real data sets to illustrate the importance and flexibility of the TIIHLW distribution. We compare the fits of the TIIHLW model with some models namely; the beta Weibull (BW) (Lee et al. (2007)), Mcdonald Weibull (McW) (Cordeiro et al. 2014) and exponentiated Weibull (EW) (Mudholkar and Srivastava (1993)) dsitributions.

The maximized log-likelihood (-21), Akaike information criterion (AIC), corrected Akaike information criterion (CAIC), Bayesian information criterion (BIC), Hannan-Quinn information criterion (HQIC), Anderson-Darling $\left(A^{*}\right)$ and Cramér-von Mises ( $\left.W^{*}\right)$ statistics are used for model selection.

\section{Example 1:}

The data have been obtained from Nicholas and Padgett (2006). The data represent tensile strength of 100 observations of carbon fibers and they are:

$3.7,3.11,4.42,3.28,3.75,2.96,3.39,3.31,3.15,2.81,1.41,2.76,3.19,1.59,2.17,3.51,1.84,1.61$, $1.57,1.89,2.74,3.27,2.41,3.09,2.43,2.53,2.81,3.31,2.35,2.77,2.68,4.91,1.57,2.00,1.17,2.17$, $0.39,2.79,1.08,2.88,2.73,2.87,3.19,1.87,2.95,2.67,4.20,2.85,2.55,2.17,2.97,3.68,0.81,1.22$, $5.08,1.69,3.68,4.70,2.03,2.82,2.50,1.47,3.22,3.15,2.97,2.93,3.33,2.56,2.59,2.83,1.36,1.84$, $5.56,1.12,2.48,1.25,2.48,2.03,1.61,2.05,3.60,3.11,1.69,4.90,3.39,3.22,2.55,3.56,2.38,1.92$, $0.98,1.59,1.73,1.71,1.18,4.38,0.85,1.80,2.12,3.65$.

For the data in Example 1, Table 3 gives the MLEs of the fitted models and their standard errors (SEs) in parenthesis. The values of goodness-of-fit statistics are listed in Table 4. 
It is noted, from Table 4, that the TIIHLW distribution provides a better fit than the other competitive fitted models. It has the smallest values for goodness-of-fit statistics among all fitted models. Plots of the histogram, fitted densities and estimated cdfs are shown in Figure 6. These figures supported the conclusion drawn from the numerical values in Table 4.

\section{Example 2:}

The second data set is obtained from Tahir et al. (2015) and represents failure times of 84 Aircraft Windshield. The data are:

$0.040,1.866,2.385,3.443,0.301,1.876,2.481,3.467,0.309,1.899,2.610,3.478,0.557,1.911$, $2.625,3.578,0.943,1.912,2.632,3.595,1.070,1.914,2.646,3.699,1.124,1.981,2.661,3.779$, $1.248,2.010,2.688,3.924,1.281,2.038,2.82,3,4.035,1.281,2.085,2.890,4.121,1.303,2.089$, $2.902,4.167,1.432,2.097,2.934,4.240,1.480,2.135,2.962,4.255,1.505,2.154,2.964,4.278$, $1.506,2.190,3.000,4.305,1.568,2.194,3.103,4.376,1.615,2.223,3.114,4.449,1.619,2.224$, $3.117,4.485,1.652,2.229,3.166,4.570,1.652,2.300,3.344,4.602,1.757,2.324,3.376,4.663$.

Based on first data, the MLEs of the fitted models and their SEs in parenthesis are listed in Table 3. Also, the values of goodness-of-fit statistics are presented in Table 4.

Table 3: The MLEs and SEs of the Model Parameters for First Data Set

\begin{tabular}{cccccc}
\hline Model & \multicolumn{5}{c}{ Estimates (SEs) } \\
TIIHLW $(\lambda, \delta, \gamma)$ & 1.025 & 0.022 & 3.15 & & \\
& $(0.13809)$ & $(0.013)$ & $(0.416)$ & & \\
$\mathbf{B W}(a, b, \lambda, \gamma)$ & 34.051 & 14.541 & 0.833 & 0.427 & \\
& $(0.961)$ & $(0.19)$ & $(0.11)$ & $(0.077)$ & \\
$\operatorname{McW}(a, b, \lambda, \gamma, c)$ & 35.28 & 18.125 & 0.813 & 0.399 & 1.548 \\
& $(0.916)$ & $(0.254)$ & $(0.13)$ & $(0.085)$ & $(6.993)$ \\
$\mathbf{E W}(\lambda, \gamma, a)$ & 5.77 & 0.295 & 1135 & & \\
& $(0.103)$ & $(0.057)$ & $(0.662)$ & & \\
\hline
\end{tabular}

Table 4: Goodness-of-Fit Statistics for First Data Set

\begin{tabular}{cccccccc}
\hline Model & -2 l & AIC & CAIC & BIC & HQIC & $A^{*}$ & $W^{*}$ \\
\hline TIIHLW & $\mathbf{2 8 8 . 1 3 4}$ & $\mathbf{2 9 4 . 1 3 4}$ & $\mathbf{2 9 4 . 3 8 4}$ & $\mathbf{2 9 4 . 1 3 4}$ & $\mathbf{2 9 7 . 2 9 7}$ & $\mathbf{0 . 4 9 2 5 2}$ & $\mathbf{0 . 0 8 7 4 5}$ \\
BW & 317.214 & 325.214 & 326.814 & 325.214 & 329.431 & 1.22496 & 0.23356 \\
McW & 308.116 & 318.116 & 319.716 & 318.116 & 323.388 & 1.22090 & 0.23286 \\
EW & 373.861 & 377.861 & 378.305 & 376.815 & 378.757 & 2.81959 & 0.51324 \\
\hline
\end{tabular}

As seen from Table 4 that the TIIHLW distribution with provides a better fit than the other competing models. It has the smallest $-2 \mathrm{l}$, AIC, CAIC, BIC, HQIC, $A^{*}$ and $W^{*}$ values among those considered here. Additionally; plots of the fitted densities and the histogram are given in Figure 6. 


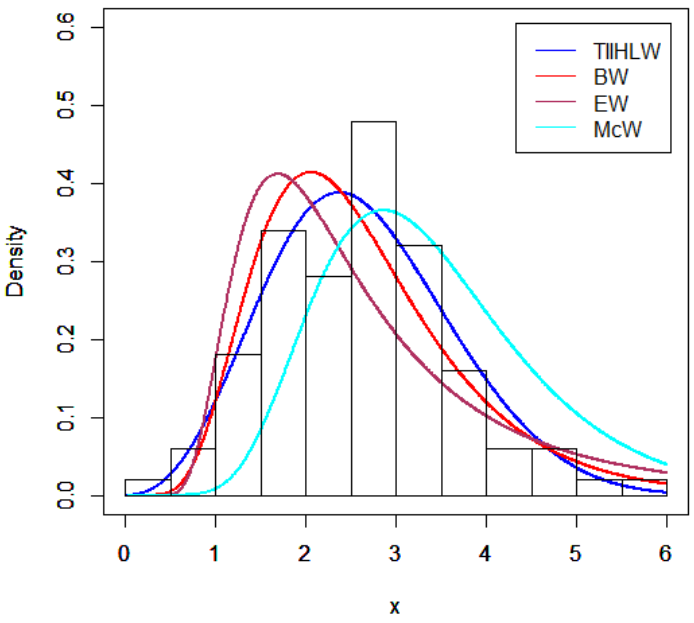

(a)

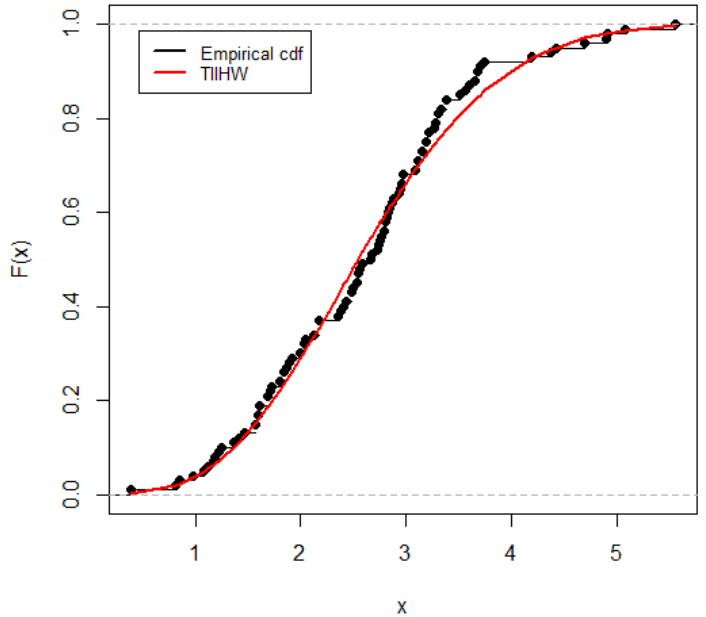

(b)

Figure 6: Estimated pdf and cdf plots for first data set

By considering the second real data, MLEs of the fitted models and their SEs in parenthesis are given in Table 5. Further, the values of goodness-of-fit statistics are presented in Table 6.

Table 5: The MLEs and SEs for Second Data Set

\begin{tabular}{cccccc}
\hline Model & \multicolumn{5}{c}{ Estimates (SEs) } \\
\hline \multirow{2}{*}{ TIIHLW $(\lambda, \delta, \gamma)$} & 0.744 & 0.015 & 3.223 & & \\
& $(0.23878)$ & $(0.021)$ & $(0.683)$ & & \\
BW $(a, b, \lambda, \gamma)$ & 53.874 & 20.528 & 1.076 & 0.231 & \\
& $(2.717)$ & $(0.278)$ & $(0.278)$ & $(0.184)$ & \\
McW $(a, b, \lambda, \gamma, c)$ & 51.321 & 19.762 & 1.119 & 0.23 & 1.525 \\
& $(5.329)$ & $(0.605)$ & $(0.48)$ & $(0.424)$ & $(38.539)$ \\
EW $(\lambda, \gamma, a)$ & 7.017 & 0.144 & 1773 & & \\
& $(0.134)$ & $(0.063)$ & $(0.827)$ & & \\
\hline
\end{tabular}

Table 6: Goodness-of-Fit Statistics for Second Data Set

\begin{tabular}{cccccccc}
\hline Model & -21 & AIC & CAIC & BIC & HQIC & $A^{*}$ & $W^{*}$ \\
\hline TIIHLW & $\mathbf{2 6 0 . 9 0 5}$ & $\mathbf{2 6 6 . 9 0 5}$ & $\mathbf{2 6 7 . 2 0 5}$ & $\mathbf{2 6 6 . 6 7 8}$ & $\mathbf{2 6 9 . 8 3 7}$ & $\mathbf{0 . 7 2 6 3 6}$ & $\mathbf{0 . 0 7 8 0 6}$ \\
BW & 289.948 & 297.948 & 298.455 & 297.645 & 301.857 & 3.34711 & 0.48715 \\
McW & 283.983 & 293.983 & 294.752 & 293.604 & 298.869 & 3.33313 & 0.4847 \\
EW & 320.347 & 326.347 & 326.647 & 324.196 & 326.302 & 32.74879 & 7.04167 \\
\hline
\end{tabular}

It is observed, from Table 6, that the TIIHLW distribution gives a better fit than other fitted models. 


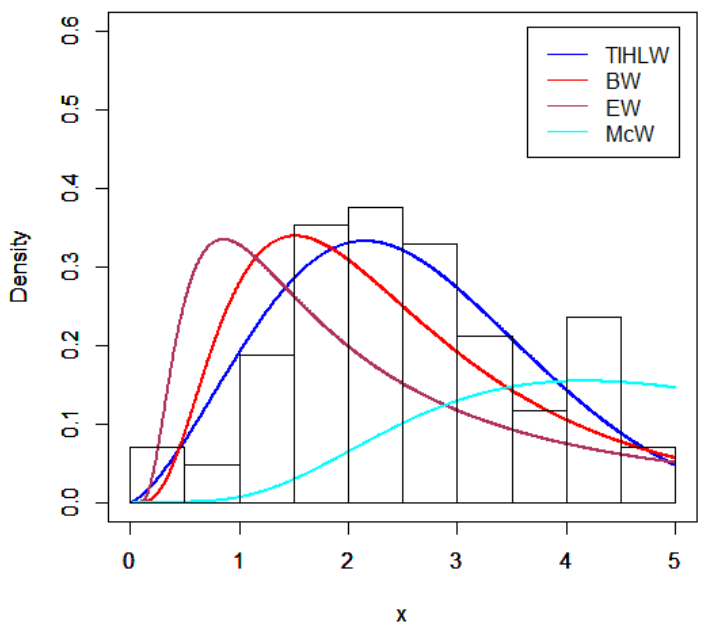

(a)

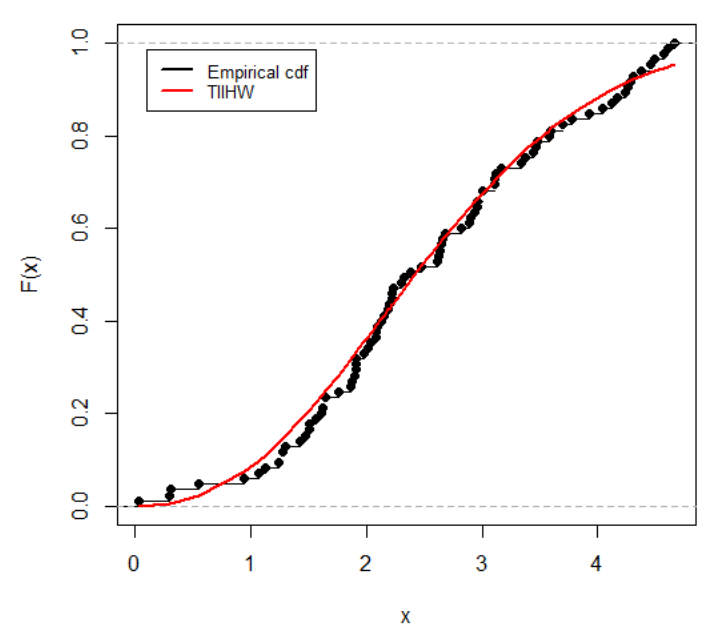

(b)

Figure 7: Estimated pdf and cdf plots for first data set

Figure 7 displays the empirical and four fitted cumulative distribution functions for the second data. The TIIHLW model provides adequate fits than the other competing models.

\section{Conclusion}

In this paper, we propose a three-parameter model, named the TIIHLW distribution. The TIIHLW model is motivated by the wide use of the Weibull distribution in practice and also for the fact that the generalization provides more flexibility to analyze positive real-life data. We derive explicit expressions for the quantile function, ordinary and incomplete moments, order statistics and Rényi entropy. The maximum likelihood estimation of the model parameters is investigated. We provide some simulation results to assess the performance of the proposed model. The practical importance of the TIIHLW distribution is demonstrated by means of two data sets.

\section{References}

[1] Alexander, C., Cordeiro, G. M., Ortega, E.M.M. and Sarabia, J. M. (2012). Generalized beta generated distributions. Computational Statistics and Data Analysis, 56: 1880-1897.

[2] Alizadeh, M., Emadi, M., Doostparast, M., Cordeiro, G.M., Ortega, E.M.M., and Pescim, R.R. (2015). Kumaraswamy odd log-logistic family of distributions: Properties and applications. Hacettepe Journal of Mathematics and Statistics, 44(6): 1491-1512.

[3] Alzaatreh, A., Lee, C. and Famoye, F. (2013). A new method for generating families of continuous distributions. Metron, 71, 63-79.

[4] Carrasco M., Ortega E. M., and Cordeiro G. M. (2008). A generalized modified Weibull distribution for lifetime modeling. Computational Statistics and Data Analysis, 53: 450-462.

[5] Cordeiro, G. M. and de Castro, M. (2011). A new family of generalized distribution. Journal of Statistical Computations and Simulation, 81: 883-898.

[6] Cordeiro, G. M., Hashimoto, E. M., and Ortega, E. M.M. (2014).The McDonald Weibull model. A Journal of Theoretical and Applied Statistics 48(2): 256-278.

[7] Cordeiro, G. M., Alizadeh, M. and Marinho, E. P. R. D. (2015). The type I half-logistic family of distributions. Journal of Statistical Computation and Simulation, 86: 707-728. 
[8] David, H. A. (1981). Order Statistics, Second edition. Wiley, New York.

[9] Elgarhy, M., Hassan, A.S., and Rashed, M. (2016). Garhy-generated family of distributions with application. Mathematical Theory and Modeling, 6(2): 1-15.

[10] Eugene, N., Lee, C. and Famoye, F. (2002). The beta-normal distribution and its applications. Communications in Statistics \& Theory and Methods, 31: 497-512.

[11] Greenwood, J.A., Landwehr, J.M., and N.C. Matalas, (1979). Probability weighted moments: Definitions and relations of parameters of several distributions expressible in inverse form. Water Resources Research, 15: 1049-1054.

[12] Hassan, A. S. and Elgarhy, M. (2016 a). Kumaraswamy Weibull-generated family of distributions with applications. Advances and Applications in Statistics, 48: 205-239.

[13] Hassan, A.S. and Elgarhy, M., (2016 b). A new family of exponentiated Weibull-generated distributions. International Journals of Mathematics and Its Applications, 4(1): 135-148.

[14] Hassan, A. S., and Hemeda, S. E. (2016). The additive Weibull-G family of probability distributions. International Journals of Mathematics and Its Applications. 4(2): 151-164.

[15] Hassan, A. S., and Nassr, S. G. (2018). Power Lindley-G family. Annals of Data Science, 1-22.

[16] Hassan, A. S., Elgarhy, M., and Shakil, M. (2017a). Type II half Logistic family of distributions with applications. Pakistan Journal of Statistics \&Operation Research, 13(2): 245-264.

[17] Hassan, A. S., Hemeda, S. E., Maiti, S.S. and Pramanik, S. (2017 b). The generalized additive WeibullG family of probability distributions. International Journal of Statistics and Probability, 6(5): 65-83.

[18] Kenney, J. F. and Keeping, E. (1962). Mathematics of Statistics. D. Van Nostr and Company.

[19] Lee, C., Famoye, F. and Olumolade, O. (2007). Beta-Weibull distribution: Some properties and applications to censored data. Journal of Modern Applied Statistical Methods, 6: 173-186.

[20] Moors, J. J. A. (1988). A quantile alternative for kurtosis. Journal of the Royal Statistical Society. Series D (The Statistician), 37(1): 25-32.

[21] Mudholkar, G. S. and Srivastava, D. K. (1993). Exponentiated Weibull family for analysing bathtub failure rate data. IEEE Transactions on Reliability, 42: 299-302.

[22] Mudholkar, G.S., Srivastava, D.K., and Kollia, G.D. (1996). A generalization of the Weibull distribution with application to the analysis of survival data, Journal of the American Statistical Association, 91: 15751583.

[23] Nicholas, M. D. and Padgett, W. J. (2006). A bootstrap control chart for Weibull percentiles. Quality and Reliability Engineering International, 22: 141-151.

[24] Rényi A. (1961). On measures of entropy and information. In: Proceedings of the $4^{\text {th }}$ Fourth Berkeley Symposium on Mathematical Statistics and Probability, 547-561. University of California Press, Berkeley.

[25] Tahir, M. H., Cordeiro, G. M., Mansoor, M. and Zubair, M. (2015). The Weibull-Lomax distribution: Properties and applications. Hacettepe Journal of Mathematics and Statistics, 44: 461-480.

[26] Xie, M., Tang, Y., and Goh T. N. (2002). A modified Weibull extension with bathtub-shaped failure rate function. Reliability Engineering System Safety, 76: 279-85.

[27] Zografos, K. and Balakrishnan, N. (2009). On families of beta- and generalized gamma-generated distributions and associated inference. Statistical Methodology, 6: 344- 362. 